\title{
Adaptive multi-polling scheduler for QoS support of video transmission in IEEE 802.11e WLANs
}

\begin{abstract}
The 802.11E Task Group has been established to enhance quality of service (QoS) provision for time-bounded services in the current IEEE 802.11 medium access control protocol. The QoS is introduced throughout hybrid coordination function controlled channel access (HCCA) for the rigorous QoS provision. In HCCA, the station is allocated a fixed transmission opportunity (TXOP) based on its TSPEC parameters so that it is efficient for constant bit rate streams. However, as the profile of variable bit rate traffics is inconstant, they are liable to experience a higher delay especially in bursty traffic case. In this paper, we present a dynamic TXOP assignment algorithm called adaptive multi-polling TXOP scheduling algorithm (AMTXOP) for supporting the video traffics transmission over IEEE 802.11e wireless networks. This scheme invests a piggybacked information about the size of the subsequent video frames of the uplink streams to assist the hybrid coordinator accurately assign the TXOP according to actual change in the traffic profile. The proposed scheduler is powered by integrating multi-polling scheme to further reduce the delay and polling overhead. Extensive simulation experiments have been carried out to show the efficiency of the AMTXOP over the existing schemes in terms of the packet delay and the channel utilization.
\end{abstract}

Keyword: HCCA; IEEE 802.11e; Multi-polling; Quality of service; TXOP; WLAN 